\title{
08SG2/OsBAK1 regulates grain size and number, and functions differently in Indica and Japonica backgrounds in rice
}

Hua Yuan ${ }^{1,2+}$, Shijun Fan ${ }^{1,2+}$, Juan Huang ${ }^{1,3}$, Shijie Zhan ${ }^{1,2}$, Shifu Wang ${ }^{1,2}$, Peng Gao ${ }^{1,2}$, Weilan Chen ${ }^{1,2}$, Bin Tu Tu $^{1,2}$ Bingtian Ma ${ }^{1,2}$, Yuping Wang ${ }^{1,2}$, Peng Qin ${ }^{1,2^{*}}$ and Shigui $\mathrm{Li}^{1,2^{*}}$

\begin{abstract}
Background: Both grain size and grain number are significant for rice yield. In the past decade, a number of genes related to grain size and grain number have been documented, however, the regulatory mechanisms underlying them remains ambiguous.

Results: We identified a rice small grain (sg2) mutant in an EMS mutant library generated from an indica variety, Shuhui498. Using the MutMap gene mapping strategy, we identified two linkage regions on chromosome 7 and 8 , respectively, consistent with the segregation ratios in the $F_{2}$ population. We focused on the linkage region on chromosome 8, and named this locus as 08592. One of three SNPs identified in the linkage region was located in an exon of OsBAK1, leading to a nonsynonymous mutation in the kinase domain. The plant harboring the mutant version 08sg2 locus exhibited a decreased grain size, grain number and plant height. Cytological analysis indicated that $085 \mathrm{~S} 2$ regulated spikelet hull development by affecting cell proliferation. The grain size and number of knockout mutants of OsBAK1 in the japonica background were significantly decreased, but less so than in 08sg2, supporting the idea that the SNP in OsBAK1 was responsible for the 08sg2 phenotype, but that 08SG2/OsBAK1 function differently in indica and japonica backgrounds. 08sg2 was insensitive to 24-epiBL, and the expression of BR-related genes was obviously altered in 08sg2. The proportionally decreased grain length when 08sg2 and GS3 were combined indicate that 08SG2 and GS3 regulate grain length independently.

Conclusions: Our work shows that 08SG2/OsBAK1 is important for rice yield in both indica and japonica backgrounds, by regulating grain size and grain number, and the function of 08SG2/OsBAK1 is obviously affected by genetic background. The amino acid substituted in $08 \mathrm{sg} 2$ is highly conserved among different species, supporting the idea that it is important for the molecular function of 085G2/OSBAK1. Together, our work is helpful for fully understanding the function of 08SG2/OsBAK1.
\end{abstract}

Keywords: Rice, 08SG2/OsBAK1, Grain size, Grain number, BR, Cell proliferation

\section{Background}

Grain size and grain number are the most important factors determining grain yield in rice. So far, a lot of genes related to grain size and number have been documented. Regarding grain size, multiple signaling pathways that influence grain size have been identified in rice $(\mathrm{Li}$ and Li 2014, 2016; Zheng et al. 2015; Zuo and Li 2014),

\footnotetext{
* Correspondence: qinpeng626@gmail.com; lishigui@sicau.edu.cn ${ }^{\dagger}$ Equal contributors

${ }^{1}$ Rice Research Institute of Sichuan Agricultural University, Chengdu Wenjiang, Sichuan 611130, China

Full list of author information is available at the end of the article
}

including the ubiquitination-mediated proteasomal degradation pathway, G-protein signaling pathway, the mitogenactivated protein kinase signaling pathway, transcriptional regulatory factors and phytohormone pathways. The ubiquitination-mediated proteasomal degradation pathway includes GW2 (Song et al. 2007) and GW5/qSW5 (Shomura et al. 2008; Weng et al. 2008), and the Gprotein signaling pathway includes GS3 and DEP1. GS3 is a homolog of $A G G 3$, the G protein $\gamma$-subunit of Arabidopsis ( $\mathrm{Li}$ et al. 2012), which encodes a putative transmembrane protein and functions as a negative regulator for 
grain size (Fan et al. 2006; Mao et al. 2010). DEP1 encodes a plant-specific $G$ protein $\gamma$ subunit and mutants in it have short grains (Huang et al. 2009). The mitogenactivated protein kinase (MAPK) signaling pathway includes OsMKK4 and OsMAPK6. OsMAPK6 interacts strongly with OsMKK4, indicating that the OsMKK4OsMAPK6 cascade influences grain size in rice (Duan et al. 2014; Liu et al. 2015). The transcriptional regulatory factors pathway includes GW8, GLW7 and GS2/GL2/GLW2/ OsGRF4. GW8 and GLW7 encode the plant-specific transcription factors OsSPL16 and OsSPL13 (Si et al. 2016; Wang et al. 2012), respectively. GS2/GL2/GLW2 encodes a transcription factor Growth-Regulating Factor 4 (OsGRF4), which regulates grain length and width mainly by promoting cell expansion (Che et al. 2015; Duan et al. 2015; Hu et al. 2015; Li et al. 2016). Another important signaling pathway controlling grain size is phytohormone pathway, which has been proven to play a crucial role in rice development, especially in the course of grain growth. For example, GS5, a putative serine carboxypeptidase, functions as a positive regulator of grain size ( $\mathrm{Li}$ et al. 2011), and inhibits the interaction between OsBAK1-7 and OsMSBP1, suggesting that GS5 might regulate grain size through the brassinosteroids (BR) signaling pathway (Xu et al. 2015). GL3.1/qGL3 encodes a putative protein phosphatase with a Kelch-like repeat domain (OsPPKL1), and may function as a negative regulator through BR signaling in rice (Hu et al. 2012; Qi et al. 2012; Zhang et al. 2012). Regarding grain number, many QTLs and genes have been cloned, such as Gn1a (Ashikari et al. 2005), DEP1 (Yan et al. 2007), DEP3 (Qiao et al. 2011), GNP1 (Wu et al. 2016b) and GAD1 (Jin et al. 2016). However, as complex agronomic traits, the regulatory mechanism of grain size and number remains largely unknown.

OsBAK1, as a BRI1-associated receptor kinase (BAK1), has been proven to participate in BR signal transduction and regulate plant architecture in rice ( $\mathrm{Li}$ et al. 2009; Shimin et al. 2014), but no mutants have been identified so far, and there was no comprehensive investigation of $O s B A K 1$ function on yield-related traits. In this study, we identified a mutant of $O S B A K 1$ in the indica background, named $08 s g 2$, which exhibited a decreased plant height, grain size, grain number and panicle length. Loss of function of OsBAK1 in the japonica background exhibited significantly decreased grain size and number, but less so than in the indica background. Our results show that the reduced grain length of $08 s g 2$ was due to decreased cell proliferation. $08 s g 2$ was insensitive to BR, and 08SG2 affected the expression of BR-related genes. Pyramiding analysis indicated that 08SG2/OsBAK1 regulate grain length independently of GS3.

\section{Results}

\section{Isolation the $08 \mathrm{sg} 2$ mutant from a small grain (sg2)} mutant

We identified a small grain (sg2) mutant from an EMS mutant library generated from an indica variety Shuhui498 (R498), which is an excellent restorer of hybrid rice. The $s g 2$ mutant showed a significant change of plant height, grain size and panicle architecture (Additional file 1: Figure S1, Additional file 1: Table S1). To identify the gene responsible for the $s g 2$ phenotype, we generated a $F_{2}$ population from a cross between $s g 2$ mutant and the parental line R498. Only 28 plants, about one-sixteenth of the plants $\left(\chi_{(15: 1)}^{2}=1.45<\left(\chi_{(0.05,1)}^{2}=3.84\right)\right.$ showed short grain length (Additional file 1: Figure S2A), suggesting that two recessive loci were responsible for the $s g 2$ phenotype. Subsequently, we applied the MutMap strategy for gene mapping (Abe et al. 2012). Consistent with the segregation ratio, we identified two linkage regions on chromosome 7 and 8, respectively (Additional file 1: Figure S2B). These results suggest that the $s g 2$ mutant phenotype is due to two recessive mutations, simultaneously. In this study, we focused on the linkage region on chromosome 8 , and named this locus $08 \mathrm{sg} 2$.

Three linkage SNPs (SNP index $=1$ ) on chromosome 8 were identified (Additional file 1: Table S2), which were located in LOC_Os08g05980, LOC_Os08g07760 and LOC_Os08g08000, respectively. RNA-sequencing showed that only LOC_Os08g07760 and LOC_Os08g08000 were expressed in young panicles, furthermore, only the SNP2 in LOC_Os08g07760 led to a nonsynonymous mutation of a glycine (GGT) to an aspartic acid (GAT) (Additional file 1: Table S2). Therefore, we considered LOC_Os08g07760 as the best candidate gene for 08SG2, which was identified as a BRI1-associated receptor kinase (BAK1) that participates in BR signal transduction ( $\mathrm{Li}$ et al. 2009). Then, we obtained plants harboring only the $08 \mathrm{sg} 2$ locus using markers based on the SNP in 08SG2/OsBAK1, and generated a $\mathrm{F}_{2}$ segregation population from a cross between 08sg2 and R498. The SNP in LOC_Os08g07760 was cosegregated with the plants with extremely short grain from $\mathrm{F}_{2}$ population (Additional file 1: Figure S3), confirming $L O C \_O s 08 g 07760$ as the best candidate gene for $08 S G 2$.

\section{The $08 \mathrm{sg} 2$ mutant exhibits a decreased grain size and grain number}

To eliminate other potential mutations, $08 s g 2$ was backcrossed with R498 three times. Compared with R498, the plant height of $08 \mathrm{sg} 2$ was reduced (Fig. 1a, i), which was mainly due to the shortened panicle length and uppermost internode (Additional file 1: Figure S4). The grain length and width of $08 \mathrm{sg} 2$ was reduced, $10.2 \%$ and $2.7 \%$, respectively, as a result, the 1000 -grain weight of $08 \mathrm{sg} 2$ was reduced $12.8 \%$ (Fig. 1b, c, f, g, h). We dynamically 


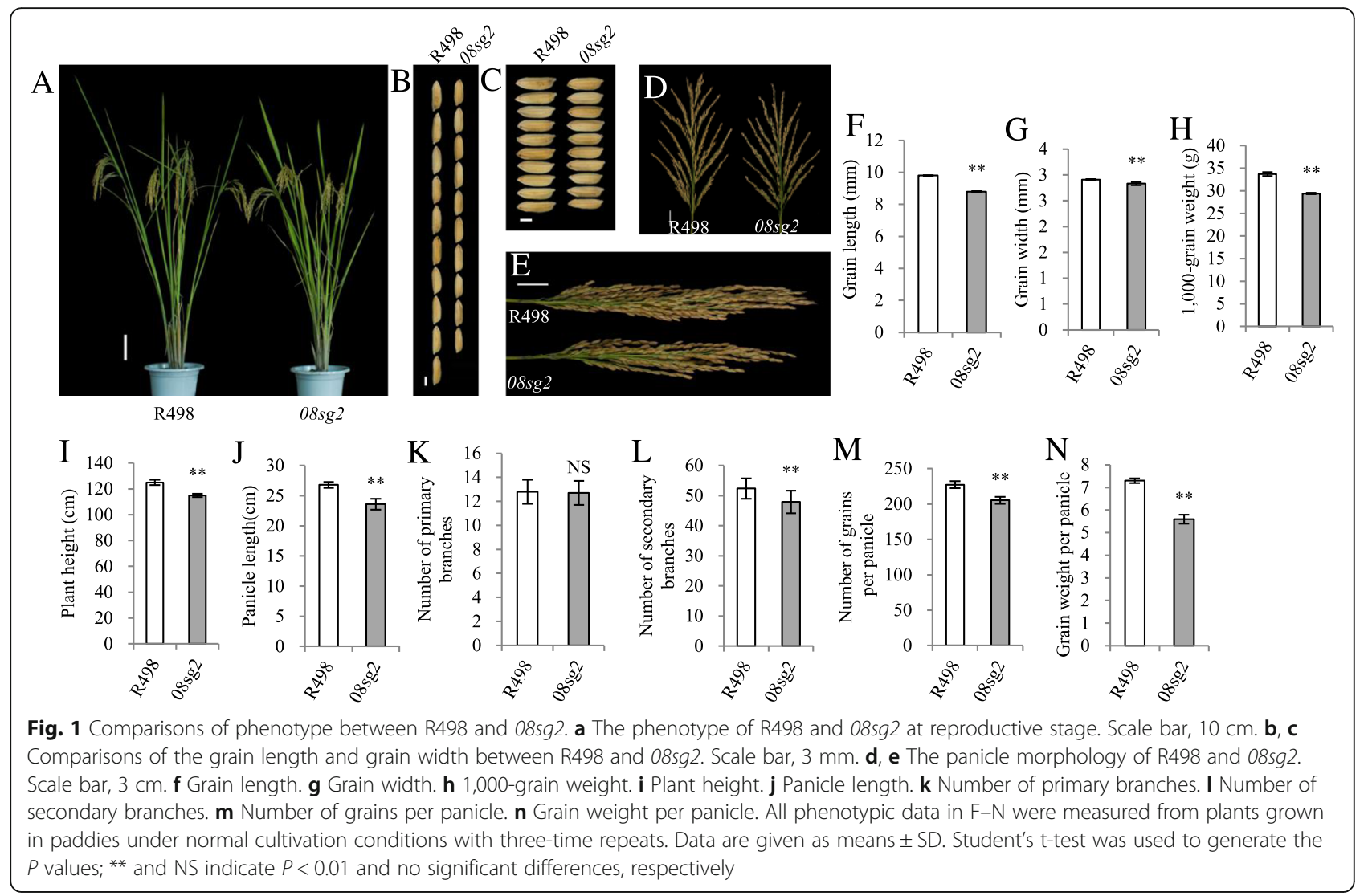

investigated the fresh and dry weight of endosperm between $08 s g 2$ and R498. Both fresh and dry weight of $08 s g 2$ endosperm was significantly less than those of R498 from 9 days after fertilization (Additional file 1: Figure S5), indicating that $08 s g 2$ had a slower grain filling rate. In addition, the panicle length of $08 s g 2$ was reduced $11.9 \%$ (Fig. 1e, j). The number of primary branches had no significant difference from that of R498 (Fig. 1d, k), but the number of secondary branches was reduced $8.6 \%$, resulting in a $9.7 \%$ reduction in grain number per panicle (Fig. 1l, m). Consistent with this, the expression of OsCLV1 and OsCLV2, which are negative regulators of panicle branching (Chu et al. 2006; Suzaki et al. 2004), were significantly up-regulated in $08 s g 2$ (Additional file 1 : Figure S6). Due to the reduction of both grain size and grain number, the grain weight per panicle of $08 s g 2$ was significantly reduced, up to $23.3 \%$ (Fig. 1n).

\section{SG2 regulates the development of spikelet hull by affecting cell proliferation}

To explore the cytological mechanism which led to the reduction of spikelet hulls, we used scanning electron microscopy (SEM) to compare the cell lengths of the inner and outer epidermal cells in spikelet hulls in R498 and $08 s g 2$ (Fig. 2b-e). The cell length of the inner and outer epidermal cells of R498 and 08sg2 were not statistically different (Fig. 2g, i), but the number of inner and outer epidermal cells in the longitudinal direction was significantly less in $08 s g 2$ than in R498 (Fig. 2h, j). Taken together, these results indicate that the reduction in cell number is responsible for the decreased grain length of $08 s g 2$. We then checked the expression level of cell-cycle related genes in R498 and 08sg2. In young panicles, the expression level of CDT2 and several MCM genes, which are involved in the G1-to-S transition, were significantly reduced in $08 s g 2$ (Fig. 2k). These results suggest that $08 S G 2$ regulates cell number by affecting cell proliferation.

Furthermore, we analyzed the expression level of several genes related to grain size. The expression of TGW6, which negatively controls grain length and thousand-grain weight (Ishimaru et al. 2013), was increased 1.8 times in 08sg2 (Additional file 1: Figure S7), consistent with the $08 s g 2$ small grain phenotype. Therefore, 08SG2 is likely to regulate grain length through $T G W 6$.

\section{$08 \mathrm{sg} 2$ is a loss of function mutant of OsBAK1}

To further confirm that the mutation in OsBAK1 was responsible for the 08sg2 phenotype, we used the CRISPR/ Cas9 genome editing system (Shan et al. 2013) to knockout OsBAK1 in Nipponbare (japonica) background. The target site was designed at the third exon of OsBAK1 


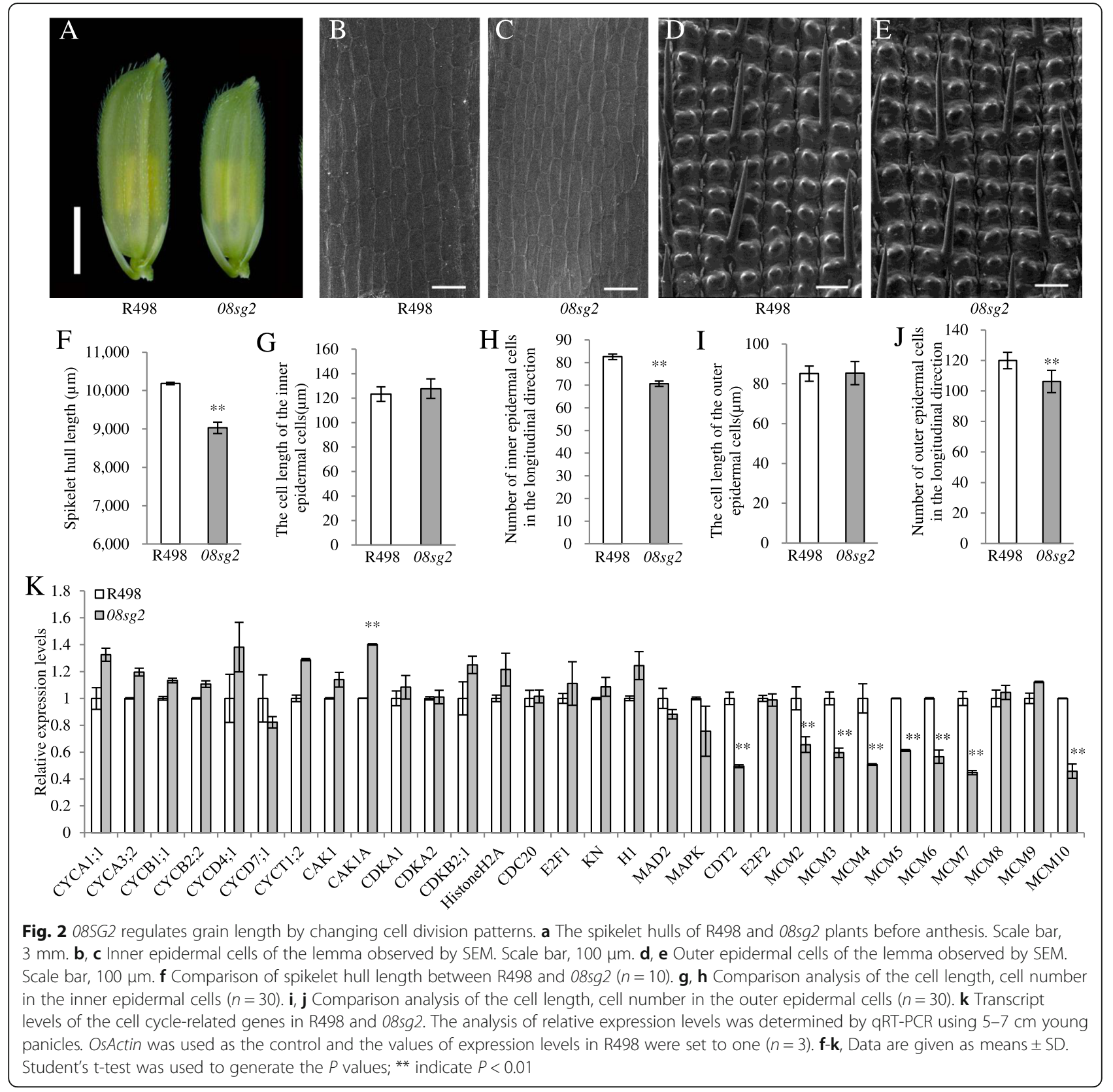

(Fig. 3a), ensuring a loss of function mutant. Four independent homozygous mutants (KO1-4) with different mutation types were obtained, and all mutations results in premature stop codons (Fig. 3b). Homozygous mutants of OsBAK1 without T-DNA insertion were obtained from $\mathrm{T}_{1}$ generation. We then comprehensively investigated the agronomic traits in the $\mathrm{T}_{2}$. Relative to WT (Nipponbare), all four loss of function lines of OsBAK1 were slightly shorter (Fig. 3i, Additional file 1: Figure S8), and the grain length and grain width decreased $6.3 \%$ and $3.4 \%$, respectively (Fig. 3c, d, f, g), resulting a $10.1 \%$ reduction of 1000-grain weight (Fig. 3h). The panicle length and grain number per panicle were reduced $7.1 \%$ and $6.0 \%$, respectively (Fig. 3e, j, k). Seed setting rate was not different (Additional file 1: Figure S9H). The reduction in both grain size and grain number resulted an $18.1 \%$ reduction of grain weight per panicle in all knockout mutants of OsBAK1 (Fig. 31). Together, OsBAK1 knockout lines in the japonica background showed significantly decreased grain size and number, as in the $08 s g 2$ mutant, but the reduction percentage was obvious smaller than 08sg2 in the indica background, except for grain width (Table 1), confirming that the SNP2 in OsBAK1 is responsible for the $08 s g 2 \mathrm{mu}$ tant phenotype, and indicating that $08 \mathrm{sg} 2$ is a loss of 


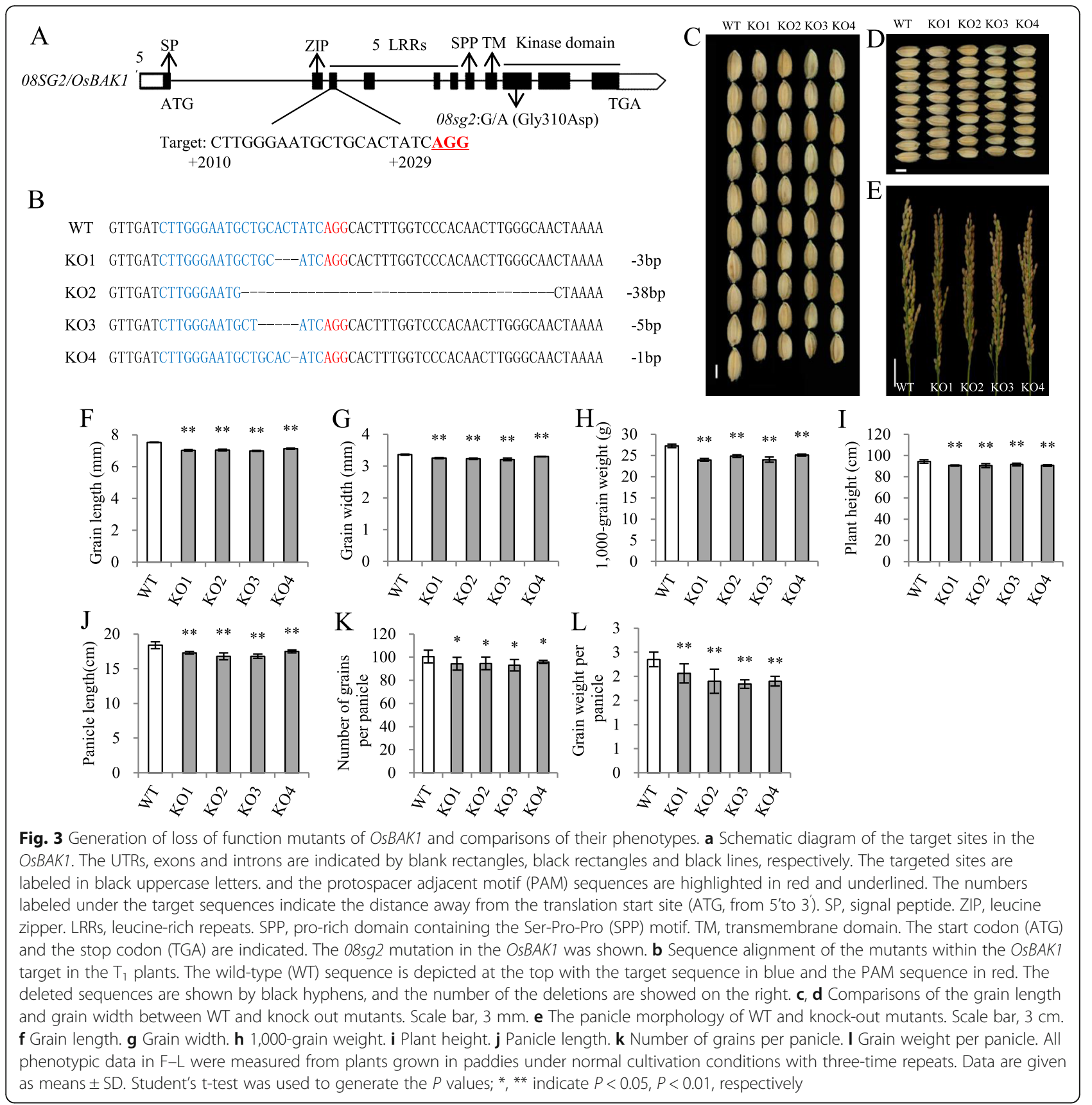

Table 1 Comparisons of the reduction percentage of 08SG2/OsBAK1 mutation in indica and japonica backgrounds

\begin{tabular}{lllllll}
\hline & Grain length & Grain width & Plant height & Panicle length & Number of grains per panicle & Grain weight per panicle \\
\hline 08sg2 vs R498 & $-10.2 \pm 0.2$ & $-2.7 \pm 0.7$ & $-8 \pm 1.1$ & $-11.9 \pm 2.8$ & $-9.7 \pm 2.1$ & $-23.3 \pm 3.1$ \\
KO vs Nipponbare & $-6.3 \pm 0.6^{* *}$ & $-3.4 \pm 1.0$ & $-3.8 \pm 0.5^{* *}$ & $-7.1 \pm 1.7^{*}$ & $-6.0 \pm 1.0^{*}$ & $-18.1 \pm 3.5^{*}$ \\
\hline
\end{tabular}

Reduction percentage (\%) was calculated using the WT (R498 and Nipponbare, respectively) as control. KO indicated the average value of the four knock out mutants. Data are given as means \pm SD. Student's t-test was used to generate the $P$ values; ${ }^{*}$ and ${ }^{* *}$ indicate $P<0.05$ and $P<0.01$, respectively 
function mutant but the effect of $08 S G 2 / O s B A K 1$ on grain size and number is different between indica and japonica background.

OsBAK1 was expressed ubiquitously in all organs examined, with a relatively higher expression in developing young inflorescences (Additional file 1: Figure S10), supporting the biological roles of $08 S G 2 / O s B A K 1$ in regulating grain size and grain number. The sequence alignment and phylogenetic analysis of the homologs of OsBAK1 showed that $O s B A K 1$ was conserved in different species (Additional file 1: Figure S11; Additional file 1: Figure S12), notably, the amino acid substitution in 08sg2 (G310D), which was located in the kinase domain of OsBAK1 (Fig. 3a), was highly conserved among different species (Additional file 1: Figure S11), suggesting that this amino acid may be important for 08SG2/OsBAK1 function.

\section{The $08 s g 2$ mutant is insensitive to 24 -epiBL}

$O s B A K 1$ was reported to participate in BR signal transduction, and its expression level affected sensitivity to BR (Li et al. 2009). Therefore, we performed a series of experiments to investigate the BR sensitivity of $08 \mathrm{sg} 2$. Firstly, we analyzed the sensitivity of the 08sg2 mutant to 24-epibrassinolide (24-epiBL) with a lamina joint assay, which is a quantitative method to analyze response to BR (Yamamuro et al. 2000). The lamina joint angles of R498 increased significantly after 24-epiBL treatment, whereas those of the $08 s g 2$ had no obvious response (Fig. 4a, e). The mesocotyl elongation of R498 was more obvious than that of $08 s g 2$ when grown in darkness (Fig. 4b, f). In addition, many studies had indicated that BR would promote coleoptile growth and inhibit root elongation with wavy form in wild type plants, a good indicator of a BR-responsive phenotype (Duan et al. 2014; Jiang et al. 2012; Liu et al. 2015; Yamamuro et al. 2000). R498 and 08sg2 seeds were germinated and grown on $1 / 2 \mathrm{MS}$ medium with or without $1 \mu \mathrm{M}$ 24-epiBL treatment, respectively. The roots of R498 exhibited a wavy, curly and reduced root length phenotype when treated with $1 \mu \mathrm{M}$ 24-epiBL, while the $08 s g 2$ showed similar growth patterns with or without exogenously supplied 24-epiBL (Fig. 4c, g). Furthermore, exogenous 24epiBL significantly promoted wild type (R498) coleoptile growth, but there was no obvious promotion in $08 \mathrm{sg} 2$ (Fig. $4 \mathrm{~d}, \mathrm{~h}$ ). Together, these results suggest that the $08 \mathrm{sg} 2$ mutant is insensitive to BR.

\section{$08 \mathrm{sg} 2$ affects the expression of BR-related genes}

08SG2/OsBAK1 was involved in BR signal transduction (Li et al. 2009) and the 08sg2 mutant appeared insensitive to BR (Fig. 4a-h). We therefore analyzed the expression level of $B R$ signaling-related genes, including OsBRI1, OsMDP1, D1, OsBZR1, OsLIC1, DLT, ILI1, BU1, RAVL1, GSK2 and TUD1 (Bai et al. 2007; Duan et al.
2006; Hu et al. 2013; Je et al. 2010; Tanaka et al. 2009; Tong et al. 2009; Tong et al. 2012; Wang et al. 2008; Wang et al. 2006; Yamamuro et al. 2000; Zhang et al. 2009). Only the expression of $B U 1$, which was reported to be a positive regulator of BR signaling, was downregulated in $08 s g 2$ (Fig. 4i).

In addition, many studies had reported that BR mutants usually had feedback regulation of BR biosynthetic genes (Duan et al. 2014; Fang et al. 2016; Hong et al. 2003; Liu et al. 2015; Yamamuro et al. 2000). We therefore analyzed the expression of BR biosynthetic genes in R498 and 08sg2 in young panicles, including OsDWARF, D2, D11, OsDWARF4 (Hong et al. 2003; Mori et al. 2002; Sakamoto et al. 2006; Tanabe et al. 2005). The expression levels of $O s D$ WARF, D2 and OsDWARF4 were significantly upregulated in $08 s g 2$ (Fig. 4i). These results suggest that mutation in $08 S G 2 / O s B A K 1$ results in the feedback regulation of $\mathrm{BR}$ biosynthesis in rice.

\section{$08 S G 2 / O s B A K 1$ and GS3 regulate grain length in independent pathways}

The major grain length QTL GS3 was reported to encode a protein with a transmembrane domain (Fan et al. 2006; Mao et al. 2010), and another study indicated that GS3 might participate in BR signaling by indirectly influencing BRS1 (Gao et al. 2015), which is involved in a BRI1-mediated BR signaling pathway in Arabidopsis thaliana (Li et al. 2001). Therefore, 08SG2/OsBAK1 might regulate grain length through GS3. The gs3 in the 08sg2 background (R498) is loss of function, which was identified by a PCR-based marker based on the functional nucleotide polymorphism in GS3 (Ramkumar et al. 2010), and exhibited a long-grain phenotype (Fig. 5a, Additional file 1: Figure S13B, D). We generated a near-isogenic line NILGS3 in the same background with $08 s g 2$, using an indica rice variety $\mathrm{R} 3551$ as the donor of functional GS3 (Additional file 1: Figure S13B, D). Compared with the grain size of R498, the grain length and width of NIL-GS3 ${ }^{\mathrm{R} 3551}$ was decreased by $11.5 \%$, and increased $1.4 \%$, respectively (Fig. 5a, b, d, e, Additional file 1: Figure S13B, C), consistent with the role of GS3 as a major QTL for grain length and a minor QTL for grain width (Fan et al. 2006). We crossed 08sg2 with NIL$G S 3^{\mathrm{R} 3551}$ and generated a pyramiding line $08 s g 2 / \mathrm{NIL}-$ $G S 3^{\mathrm{R} 3551}$. The $08 s g 2 / \mathrm{NIL}-G S 3^{\mathrm{R} 3551}$ plants showed a more severely reduced grain size; grain length and width were reduced $22.7 \%$, and increased $0.7 \%$, respectively, indicating that the effect of $08 \mathrm{sg} 2 / \mathrm{NIL}-G S 3^{\mathrm{R} 3551}$ on grain length was just a pyramiding effect of $08 s g 2 / O \operatorname{sbak} 1(-10.2 \%)$ and GS3 (-11.5\%) (Fig. 5). In addition, the 08SG2/OsBAK1 expression level was not affected in NIL-GS3 panicle, nor was the GS3 expression level in the 08sg2 mutant (Additional file 1: Figure S14). The effect of $08 \mathrm{sg} 2 /$ NIL-GS3 $3^{\text {R3551 }}$ on grain width $(0.7 \%)$ was not a simple 

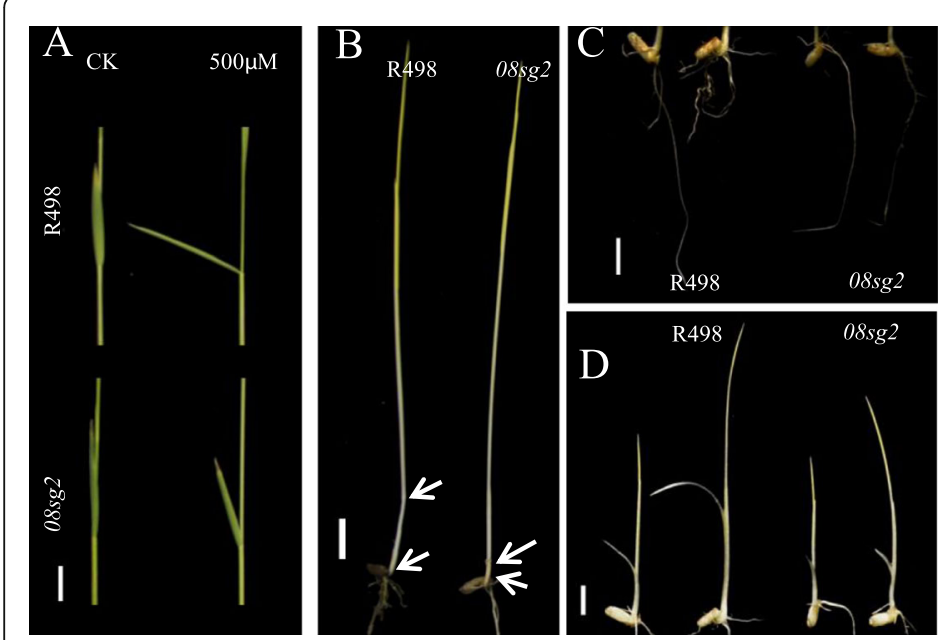

I

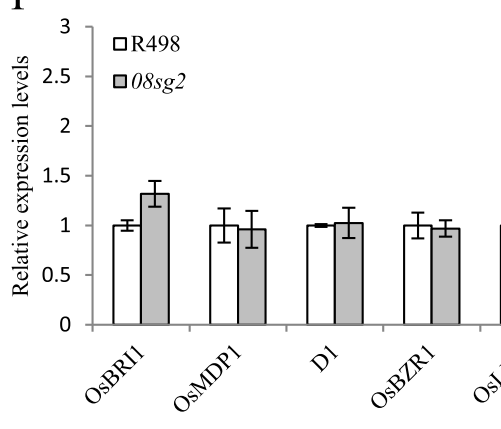

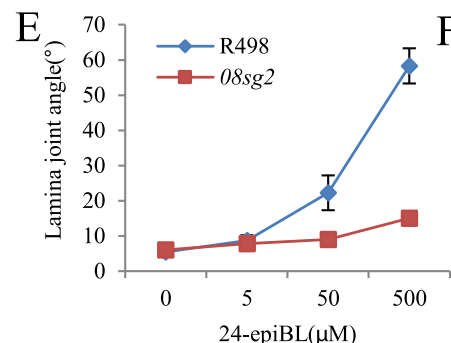
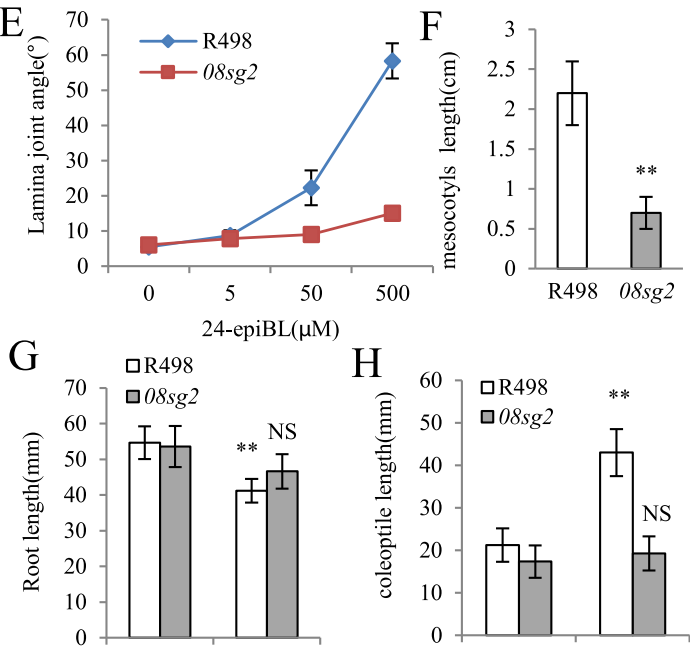

$\begin{array}{cc}0 & 1 \\ 24-\operatorname{epiBL}(\mu \mathrm{M})\end{array}$

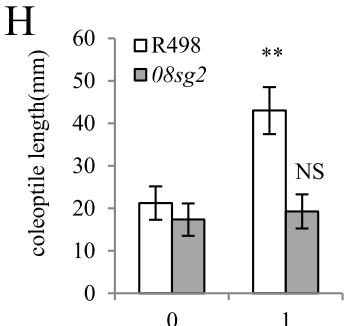
24-epiBL( $\mu \mathrm{M})$

$* *$

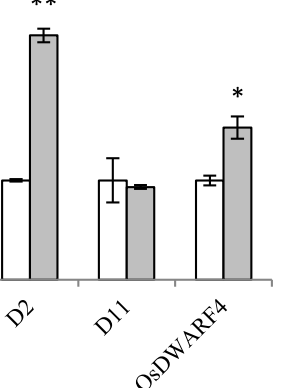

Fig. 4 The $08 s g 2$ mutant is insensitive to 24-epiBL. a Lamina joint of R498 and 08sg2 in response to 24-epiBL treatment. b Comparisons of the mesocotyls elongation between R498 and 08sg2 when plants were grown in complete darkness, arrows indicate the nodes. c Roots of R498 and 08sg2 seedlings grown on 1/2MS medium without (left) or with (right) $1 \mu \mathrm{M}$ 24-epiBL treatment. $\mathbf{d}$ The coleoptile elongation of R498 and 08 sg2 without (left) or with (right) $1 \mu \mathrm{M}$ 24-epiBL treatment. e Measurements of the lamina joints angles of the wild type and 08sg2 after treatment with various concentrations of 24-epiBL. f The mesocotyls length of R498 and 08sg2 when plants were grown in complete darkness. g Roots length of R498 and 08sg2 seedlings grown on 1/2MS medium with $1 \mu \mathrm{M}$ 24-epiBL treatment. $\mathbf{h}$ Coleoptile length of R498 and 08 sg2 seedlings grown on $1 / 2 \mathrm{MS}$ medium with $1 \mu \mathrm{M}$ 24-epiBL treatment. Bars $=10 \mathrm{~mm}$. i The analysis of relative expression level was determined by qRT-PCR using young panicles $(5-7 \mathrm{~cm})$ during the inflorescence development. OsActin was used as the control and the values of expression levels in R498 were set to one $(n=3)$. Data are given as means \pm SD. Student's t-test was used to generate the $P$ values; ${ }^{*},{ }^{*}$ and NS indicate $P<0.05, P<0.01$ and no significant differences, respectively

effect pyramiding of $08 s g 2 / O s b a k 1(-2.7 \%)$ and GS3 (1.4\%). Taken together, our results indicate that 08SG2/OsBAK1 and GS3 likely regulate grain length in independent pathways, but they might have some genetic interaction regarding grain width.

\section{Discussion}

\section{SG2/OsBAK1 plays a crucial role in the regulation of}

\section{grain size and number}

08SG2/OsBAK1, also called OsSERK1, belongs to the Somatic Embryogenesis Receptor Kinases (SERKs) family in rice (Hu et al. 2005; Ito et al. 2005). As a SERK family gene, OsBAK1 was proven to play a basic role in mediating somatic embryogenesis in rice, and might play wider roles in other organs ( $\mathrm{Hu}$ et al. 2005; Ito et al. 2005). Overexpression and knockdown of OsBAK1 showed that OsBAK1 controlled plant architecture and was involved in BR signaling ( $\mathrm{Li}$ et al. 2009; Shimin et al. 2014). But there was no comprehensive investigation of OsBAK1 function on yield-related traits, such as grain size and number, except that overexpression and knockdown of $O s B A K 1$ showed decreased grain length and increased grain width, respectively, in the ZH11 background (Li et al. 2009). However, another study showed that OsBAK1 didn't affect grain size in the Kitaake background (Shimin et al. 2014). In this study, we used loss of function mutants of $08 S G 2 / O s B A K 1$ in the indica and japonica backgrounds to investigate its genetic effect on grain size and number, and also measured other agronomic traits such as plant height, panicle length and grain weight (Fig. 1). Our results showed that the grain length and width were decreased in both indica (Shuhui498) and japonica (Nipponbare) background (Fig. 1f, g; Fig. 3f, g). These findings, different with previous reports, might be 


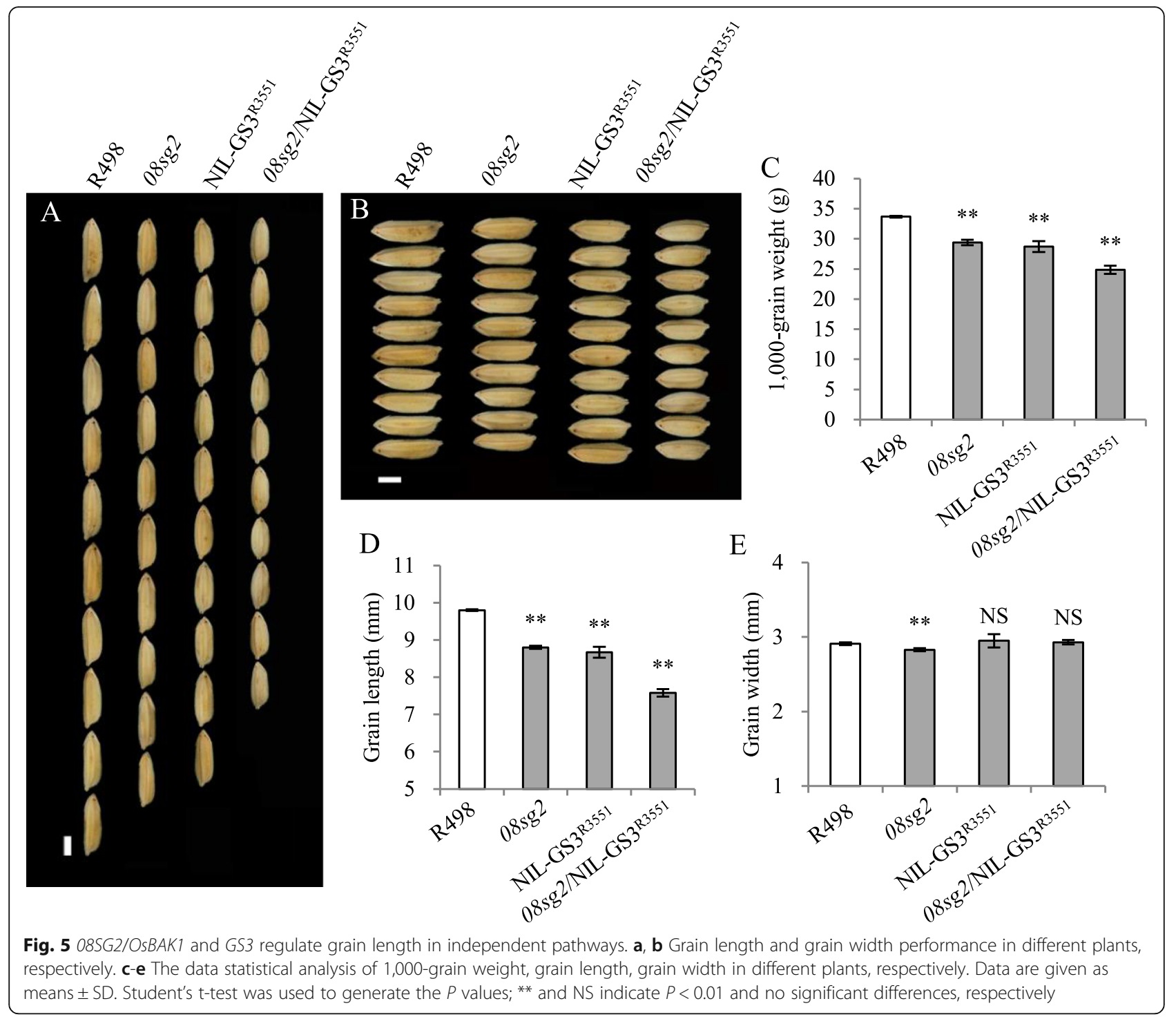

explained by ZH11 (japonica) and Kitaake (japonica) backgrounds, or because expression of OsBAK1 was not completely silenced by RNAi. Also, the decreased grain number in 08sg2/Osbak1 suggested that 08SG2/OsBAK1 is essential for rice grain number in both the indica and japonica backgrounds (Fig. 1m; Fig. 3k), which may be due to the up-regulated expression level of two negative regulators of panicle branching OsCLV1 and OsCLV2 (Additional file 1: Figure S6).

Many studies had indicated that BR-related genes regulated grain size through altering cell expansion or cell proliferation, for example: $D 2$ (Fang et al. 2016) and D11 (Wu et al. 2016a) regulated grain size by promoting cell expansion; XIAO (Jiang et al. 2012), SMG1 (Duan et al. 2014) and DSG1 (Liu et al. 2015) regulated grain size by affecting cell proliferation through cell cycle genes. Our results indicated that $08562 / O S B A K 1$ positively regulates the development of spikelet hull by affecting cell proliferation (Fig. $2 \mathrm{~h}, \mathrm{j}$ ), consistent with this notion, the expression levels of some cell-cycle related genes were downregulated in the $08 \mathrm{sg} 2$ mutant (Fig. $2 \mathrm{k}$ ), and this result is consistent with the cytological mechanism in internodes in the OsBAK1 overexpression plants (Li et al. 2009). Our results provide a new evidence that BR may control grain size via altering cell proliferation through cell cycle genes.

Even the loss of function of $085 G 2 / O s B A K 1$ showed obvious reduction on grain size and grain number in both indica (Shuhui498) and japonica (Nipponbare) backgrounds, the differences of effect on grain size and grain number were still observed at different background, for example: loss of function of $08 \mathrm{sg} 2 / \mathrm{Osbak} 1$ led to a $10.2 \%$ or $6.3 \%$ decrease in grain length, and to $9.7 \%$ or $6.0 \%$ decrease in grain number (Table 1) in indica (Shuhui498) and japonica (Nipponbare) backgrounds, respectively. This indicates that the function of $085 G 2 / O s B A K 1$ on grain size and number is affected by genetic background. 
$08 S G 2 / O s B A K 1$ regulates grain length independently with GS3

08SG2/OsBAK1 positively regulated grain length in rice. However, GS3 is a negative regulator of grain length (Fan et al. 2006). The SNP substitution in 08sg2/Osbak1 led to similar reductions in the grain length in the gs3 and GS3 background (reduced $10.20 \%$ or $12.57 \%$, respectively), and the mutation of GS3 also led to a comparable increase in the grain length in the 08sg2/Osbak1 and $08 S G 2 / O s B A K 1$ backgrounds (increased $16.09 \%$ or $13.03 \%$, respectively) (Fig. 5). In addition, the expression levels of O8SG2/OsBAK1 and GS3 were not affected by each other, suggesting that 08SG2/OsBAK1 and GS3 regulate grain length in independent pathways, with no genetic interaction.

\section{The amino acid substitution in the kinase domain of 08SG2/OsBAK1 lead to loss function}

The amino acid substitution (G310D) in 08sg2 is located in the kinase domain of OsBAK1 and this glycine is highly conserved in other species (Fig. 3a, Additional file 1: Figure S11). This amino acid substitution leads a phenotype similar to the loss of function mutant (Fig. 1; Fig. 3). OsBAK1 encodes a receptor-like protein kinase (RLK) (Ito et al. 2005). Both OsBAK1 and its homolog BAK1 in Arabidopsis are functional protein kinases capable of autophosphorylation, and a mutation at a conserved site (K329E, K330E, respectively) in the kinase domain led to loss of kinase activity (Li et al. 2002; Shimin et al. 2014). Several other studies in Arabidopsis showed that the amino acid substitution at a conserved site in the kinase domain of RLKs abolished kinase activity (Braun et al. 1997; Horn and Walker 1994), but the amino acid change in $08 s g 2$ is different. Therefore, this substituted amino acid might play a role in protein function of $08 S G 2 / O s B A K 1$, and it is worthy to investigate whether this amino acid substitution affects protein kinase activity, or other molecular functions of $08 S G 2 / O s B A K 1$.

\section{Conclusions}

08SG2/OsBAK1 plays an essential role in the regulation of grain size, grain number and plant height in both the indica and japonica backgrounds, and is important for rice yield. The function of $08 S G 2 / O s B A K 1$ on grain size and number is affected by genetic background. 08SG2/ $O s B A K 1$ regulates grain size by participating in cell proliferation, and is independent of GS3. The glycine substituted in $08 s g 2$ is located in the kinase domain, is highly conserved among different species, and is presumably important for the molecular function of 08SG2/ OsBAK1. Together, our work is helpful for unveiling the molecular function of $085 G 2 / O s B A K 1$, and indicating that $08 S G 2 / O s B A K 1$ is a potential target to manipulate for increasing rice yield.

\section{Methods}

Plant materials and growth conditions

The small grain mutant (sg2) was initially identified from an ethyl methanesulfonate (EMS) mutant population of an indica variety Shuhui498 (R498). 08sg2 was isolated from $s g 2$ in the segregation population by linkage marker and backcrossed with R498 three times. All plants were grown in paddies at the Rice Research Institute of Sichuan Agricultural University (Chengdu, China) and Hainan (Lingshui, China) under normal cultivation conditions.

\section{Cytological analysis by scanning electron microscopy (SEM)}

Spikelet hulls from $08 s g 2$ and R498 plants were collected before fertilization and fixed in $2.5 \%$ glutaraldehyde, which was prepared as described ( $\mathrm{Hu}$ et al. 2015). The fixed samples were dehydrated in a graded ethanol series (from $30 \%$ to $100 \%, 100 \%)$, followed by substitution using isopentyl acetate. The critical-point Dryer (Quorum K850, England) was used to dry samples. The samples were sputter-coated with platinum using a magnetron sputtering apparatus (JS-1600, Beijing, China). The inner and outer epidermal cells of lemma of the spikelet hulls were observed by SEM (JSM-7500 F, JEOL, Japan). Cell lengths were measured using Image J software.

\section{Gene mapping}

MutMap (Abe et al. 2012) was applied for gene mapping. Briefly, we generated an $\mathrm{F}_{2}$ population of a cross between the $s g 2$ mutant and the wild type (R498). DNA of $25 \mathrm{~F}_{2}$ progeny with small grains as $s g 2$ was extracted and bulked in an equal ratio, then this bulked DNA was subjected to whole-genome sequencing. DNA from R498 was re-sequenced as a control. The SNPs/INDELs indexes were calculated as previously described (Abe et al. 2012). We simultaneously measured allele segregation using Euclidean distance (ED) (Additional file 1: Figure S2B) as previously described (Hill et al. 2013).

\section{CRISPR/Cas9 plasmid construction, plant transformation and mutation detection}

To obtain loss of function mutants, we designed the target site at the third exon of 08SG2/OsBAK1 (Fig. 3a), then generated the CRISPR/Cas9 plasmid construct using the Biogle CRISPR/Cas kit (BGK03, http://www.biogle.cn/) according to instructions. The Oligo sequence pair (F:5''TGTGTGCTTGGGAATGCTGCACTATC-3', R:5' -AAA CGATAGTGCAGCATTCCCAAGCA-3') was generated using the online system (BGK03, http://www.biogle.cn/ index/excrispr). The final CRISPR/Cas9 plasmid construction pBGK03-OsBAK1 was introduced into Agrobacterium tumefaciens strain EHA105, and transformation of Nipponbare was performed as previously described 
(Hiei et al. 1994). For mutation detection, genomic DNA was extracted from $T_{0}$ transgenic plants and primer pairs flanking the designed target site were used for PCR amplification. PCR products were sequenced to obtain knockout plants. The sequencing primers are listed in Additional file 2: Table S3.

\section{Brassinosteroid sensitivity assay}

For lamina joint inclination analysis, after soaking and pregermination, the uniform germinated seeds of R498 and $08 s g 2$ were sown in soil and cultured in the plant growth chamber at $28{ }^{\circ} \mathrm{C}$ until two-leaf stage. $1 \mu$ of ethanol solution containing various concentrations $(0,5,50$, $500 \mu \mathrm{M})$ of 24-epiBL were dropped at the lamina joints of the second leaf of R498 and 08sg2. After cultured for 3 days, the angles between the lamina and its leaf sheath were photographed and measured. 30 plants were measured for each treatment with three replications.

For the mesocotyl elongation assay, after soaking and pre-germination, the uniform germinated seeds of R498 and $08 s g 2$ were sown in soil and cultured in the plant growth chamber at $28{ }^{\circ} \mathrm{C}$ in complete darkness for about 12 days. The mesocotyls of R498 and $08 s g 2$ were photographed and measured. 30 plants were measured for each treatment with three replications.

For the root elongation inhibition and coleoptile elongation assays, seeds of $\mathrm{R} 498$ and $08 \mathrm{sg} 2$ were dehusked, and sterilized by washing the seeds as follows: $75 \%$ ethanol for $1 \mathrm{~min}$, sterilized water for five times, $2 \%$ $\mathrm{NaClO}$ for $30 \mathrm{~min}$, then rinsed several times with sterilized water. The seeds were then germinated on halfstrength Murashige and Skoog medium supplemented with $0,1 \mu \mathrm{M} 24-\mathrm{epiBL}$ at $28{ }^{\circ} \mathrm{C}$. About a week later, root and coleoptile were photographed and lengths measured. 30 plants were measured for each treatment with three replications.

\section{RNA extraction, CDNA synthesis and quantitative real- time RT-PCR}

Total RNA was extracted from young panicles of R498, 08sg2, NIL-GS3 ${ }^{\mathrm{R} 3551}$ using Plant RNA Kit according to the product manual (OMEGA). To obtain cDNA, about $500 \mathrm{ng}$ of the total RNA was used for a genomic DNA elimination reaction and a reverse-transcription reaction using PrimeScript ${ }^{\mathrm{TM}}$ RT Reagent Kit with gDNA Eraser according to the product manual ( $\mathrm{TaKaRa})$. Quantitative realtime RT-PCR analysis was performed on the CFX96 ${ }^{\mathrm{TM}}$ Real-Time PCR system (Bio-Rad) with KAPA SYBR ${ }^{\circ}$ FAST qPCR Kit (KAPA). Actin was used as an internal control in all analyses. Three biological replicates were performed for each gene. The primers used in quantitative real-time RTPCR are listed in Additional file 2: Table S4.

\section{Additional files}

\begin{abstract}
Additional file 1: Figure S1. Morphology of wild type (R498) and sg2 mutant. Figure S2. Identification of the causal SNP of the small grain (sg2) mutant using MutMap approach. Figure S3. Linkage analysis of 08sg2. Figure S4. The 08sg2 mutant exhibited slightly shorter plant height because of the reduced panicle and the uppermost internode. Figure S5. Effect of 08SG2 on endosperm size and grain filling. Figure S6. Comparisons of transcripts of genes determining panicle branching in R498 and $08 \mathrm{sg} 2$. Figure $\mathbf{S 7}$. Comparisons of transcripts of genes determining grain size in R498 and 08sg2. Figure S8. Comparisons of phenotypes of wild type (WT, Nipponbare) and knock out (KO) mutants at reproductive stage. Figure S9. Data statistics of the other agronomic traits in R498 and 08sg2, the wild type (WT) and knock-out (KO) mutants. Figure S10. The expression pattern of 08SG2/OsBAK1 in different tissues. Figure S11. Sequence alignment of 08SG2/OsBAK1 and its orthologs in plants. Figure S12. Phylogenetic and protein similarity analysis of 085G2/ OSBAK1 orthologs in plants. Figure S13. The donor parent of GS3 has significantly smaller grain size than R498. Figure S14. 08SG2 and GS3 don't interact at transcriptional level. Table S1. Agronomic traits of R498 and sg2. Table S2. A cluster of three SNPs with SNP index of 1 on chromosome 8 (PPTX $2792 \mathrm{~kb}$ )
\end{abstract}

Additional file 2: Table S3. Primers used for PCR detection. Table S4. Primers used for qPCR analysis (XLS $58 \mathrm{~kb}$ )

\section{Acknowledgements}

We thank Hang He and Wei Yan at Peking University for MutMap analysis, and Chengzhi Liang and Qiang Gao at Institute of Genetics and Development Biology, CAS for RNA-seq analysis, and Dr. Sheila McCormick at UC Berkeley for editing.

\section{Funding}

This work was supported by grants from the National Natural Science Foundation of China (91535102 and 31471475), and the Breeding of New Varieties of Green Super Rice (2014AA10A604).

\section{Authors' Contributions}

$\mathrm{PQ}$ and $\mathrm{BT}$ generated the ethyl methanesulfonate (EMS) population. PQ, SF and $\mathrm{JH}$ identified the $s g 2$ mutant and generated the $\mathrm{F}_{2}$ population. $\mathrm{HY}$ and SF isolated the $08 s g 2$ mutant and investigated the agronomic traits. SZ, YW and PG performed the cytological analysis and performed the brassinosteroid sensitivity test. SW and BM performed quantitative real-time RT-PCR. HY and WC performed the plasmid construction, plant transformation and mutation detection. HY and SF wrote the manuscript and equally contributed to this work. PQ and SL designed and guided this research. All authors approved the manuscript.

\section{Competing Interests}

The authors declare that they have no competing interests.

\section{Publisher's Note}

Springer Nature remains neutral with regard to jurisdictional claims in published maps and institutional affiliations.

\section{Author details \\ ${ }^{1}$ Rice Research Institute of Sichuan Agricultural University, Chengdu Wenjiang, Sichuan 611130, China. ${ }^{2}$ State Key Laboratory of Hybrid Rice, Sichuan Agricultural University, Chengdu Wenjiang, Sichuan 611130, China. ${ }^{3}$ Institute of Characteristic Crops Research, Chongqing Academy of Agricultural Sciences, Yongchuan, Chongqing 402160, China.}

Received: 13 April 2017 Accepted: 18 May 2017

Published online: 25 May 2017

References

Abe A, Kosugi S, Yoshida K, Natsume S, Takagi H, Kanzaki H, Matsumura H, Yoshida K, Mitsuoka C, Tamiru M, Innan H, Cano L, Kamoun S, Terauchi R (2012) Genome sequencing reveals agronomically important loci in rice using MutMap. Nat Biotechnol 30:174-178 
Ashikari M, Sakakibara H, Lin S, Yamamoto T, Takashi T, Nishimura A, Angeles ER, Qian Q, Kitano H, Matsuoka M (2005) Cytokinin oxidase regulates rice grain production. Science 309:741-745

Bai MY, Zhang LY, Gampala SS, Zhu SW, Song WY, Chong K, Wang ZY (2007) Functions of OsBZR1 and 14-3-3 proteins in brassinosteroid signaling in rice. Proc Natl Acad Sci U S A 104:13839-13844

Braun DM, Stone JM, Walker JC (1997) Interaction of the maize and Arabidopsis kinase interaction domains with a subset of receptor-like protein kinases: implications for transmembrane signaling in plants. Plant J 12:83-95

Che R, Tong H, Shi B, Liu Y, Fang S, Liu D, Xiao Y, Hu B, Liu L, Wang H (2015) Control of grain size and rice yield by GL2-mediated brassinosteroid responses. Nat Plants 2:15195

Chu H, Qian Q, Liang W, Yin C, Tan H, Yao X, Yuan Z, Yang J, Huang H, Luo D, Ma H, Zhang D (2006) The floral organ number4 gene encoding a putative ortholog of Arabidopsis CLAVATA3 regulates apical meristem size in rice. Plant Physiol 142:1039-1052

Duan K, Li L, Hu P, Xu SP, Xu ZH, Xue HW (2006) A brassinolide-suppressed rice MADS-box transcription factor, OsMDP1, has a negative regulatory role in BR signaling. Plant J 47:519-531

Duan P, Rao Y, Zeng D, Yang Y, Xu R, Zhang B, Dong G, Qian Q, Li Y (2014) SMALL GRAIN 1, which encodes a mitogen-activated protein kinase kinase 4, influences grain size in rice. Plant J 77:547-557

Duan P, Ni S, Wang J, Zhang B, Xu R, Wang Y, Chen H, Zhu X, Li Y (2015) Regulation of OsGRF4 by OsmiR396 controls grain size and yield in rice. Nat Plants 2:15203

Fan C, Xing Y, Mao H, Lu T, Han B, Xu C, Li X, Zhang Q (2006) GS3, a major QTL for grain length and weight and minor QTL for grain width and thickness in rice, encodes a putative transmembrane protein. Theor Appl Genet 112: 1164-1171

Fang N, Xu R, Huang L, Zhang B, Duan P, Li N, Luo Y, Li Y (2016) SMALL GRAIN 11 Controls Grain Size, Grain Number and Grain Yield in Rice. Rice (N Y) 9:64

Gao X, Zhang X, Lan H, Huang J, Wang J, Zhang H (2015) The additive effects of GS3 and qGL3 on rice grain length regulation revealed by genetic and transcriptome comparisons. BMC Plant Biol 15:156

Hiei Y, Ohta S, Komari T, Kumashiro T (1994) Efficient transformation of rice (Oryza sativa L.) mediated by Agrobacterium and sequence analysis of the boundaries of the T-DNA. Plant J 6:271-282

Hill JT, Demarest BL, Bisgrove BW, Gorsi B, Su YC, Yost HJ (2013) MMAPPR: mutation mapping analysis pipeline for pooled RNA-seq. Genome Res 23: 687-697

Hong Z, Ueguchi-Tanaka M, Umemura K, Uozu S, Fujioka S, Takatsuto S, Yoshida S, Ashikari M, Kitano H, Matsuoka M (2003) A rice brassinosteroid-deficient mutant, ebisu dwarf ( $\mathrm{d} 2)$, is caused by a loss of function of a new member of cytochrome P450. Plant Cell 15:2900-2910

Horn MA, Walker JC (1994) Biochemical properties of the autophosphorylation of RLK5, a receptor-like protein kinase from Arabidopsis thaliana. Biochim Biophys Acta 1208:65-74

Hu H, Xiong L, Yang Y (2005) Rice SERK1 gene positively regulates somatic embryogenesis of cultured cell and host defense response against fungal infection. Planta 222:107-117

Hu Z, He H, Zhang S, Sun F, Xin X, Wang W, Qian X, Yang J, Luo X (2012) A Kelch motif-containing serine/threonine protein phosphatase determines the large grain QTL trait in rice. J Integr Plant Biol 54:979-990

Hu X, Qian Q, Xu T, Zhang Y, Dong G, Gao T, Xie Q, Xue Y (2013) The U-box E3 ubiquitin ligase TUD1 functions with a heterotrimeric $G$ alpha subunit to regulate Brassinosteroid-mediated growth in rice. PLoS Genet 9:e1003391

Hu J, Wang Y, Fang Y, Zeng L, Xu J, Yu H, Shi Z, Pan J, Zhang D, Kang S (2015) A rare allele of GS2 enhances grain size and grain yield in rice. Mol Plant 8: $1455-1465$

Huang X, Qian Q, Liu Z, Sun H, He S, Luo D, Xia G, Chu C, Li J, Fu X (2009) Natural variation at the DEP1 locus enhances grain yield in rice. Nat Genet 41:494-497

Ishimaru K, Hirotsu N, Madoka Y, Murakami N, Hara N, Onodera H, Kashiwagi T, Ujiie K, Shimizu B, Onishi A, Miyagawa H, Katoh E (2013) Loss of function of the IAA-glucose hydrolase gene TGW6 enhances rice grain weight and increases yield. Nat Genet 45:707-711

Ito Y, Takaya K, Kurata N (2005) Expression of SERK family receptor-like protein kinase genes in rice. Biochim Biophys Acta 1730:253-258

Je BI, Piao HL, Park SJ, Park SH, Kim CM, Xuan YH, Park SH, Huang J, Do Choi Y, An G, Wong HL, Fujioka S, Kim MC, Shimamoto K, Han CD (2010) RAV-Like1 maintains brassinosteroid homeostasis via the coordinated activation of BRI1 and biosynthetic genes in rice. Plant Cell 22:1777-1791
Jiang Y, Bao L, Jeong SY, Kim SK, Xu C, Li X, Zhang Q (2012) XIAO is involved in the control of organ size by contributing to the regulation of signaling and homeostasis of brassinosteroids and cell cycling in rice. Plant J 70:398-408

Jin J, Hua L, Zhu Z, Tan L, Zhao X, Zhang W, Liu F, Fu Y, Cai H, Sun X, Gu P, Xie D, Sun C (2016) GAD1 Encodes a Secreted Peptide That Regulates Grain Number, Grain Length, and Awn Development in Rice Domestication. Plant Cell 28:2453-2463

Li N, Li Y (2014) Ubiquitin-mediated control of seed size in plants. Front Plant Sci 5:332

Li N, Li Y (2016) Signaling pathways of seed size control in plants. Curr Opin Plant Biol 33:23-32

Li J, Lease KA, Tax FE, Walker JC (2001) BRS1, a serine carboxypeptidase, regulates BRI1 signaling in Arabidopsis thaliana. Proc Natl Acad Sci U S A 98:5916-5921

Li J, Wen J, Lease KA, Doke JT, Tax FE, Walker CJ (2002) BAK1, an Arabidopsis LRR Receptor-like Protein Kinase, Interacts with BRI1 and Modulates Brassinosteroid Signaling. Cell 110:213-222

Li D, Wang L, Wang M, Xu YY, Luo W, Liu YJ, Xu ZH, Li J, Chong K (2009) Engineering OsBAK1 gene as a molecular tool to improve rice architecture for high yield. Plant Biotechnol J 7:791-806

Li Y, Fan C, Xing Y, Jiang Y, Luo L, Sun L, Shao D, Xu C, Li X, Xiao J, He Y, Zhang Q (2011) Natural variation in GS5 plays an important role in regulating grain size and yield in rice. Nat Genet 43:1266-1269

Li S, Liu W, Zhang X, Liu Y, Li N, Li Y (2012) Roles of the Arabidopsis G protein Y subunit AGG3 and its rice homologs GS3 and DEP1 in seed and organ size control. Plant Signal Behav 7:1357-1359

Li S, Gao F, Xie K, Zeng X, Cao Y, Zeng J, He Z, Ren Y, Li W, Deng Q, Wang S, Zheng A, Zhu J, Liu H, Wang L, Li P (2016) The OsmiR396c-OsGRF4-OsGIF1 regulatory module determines grain size and yield in rice. Plant Biotechnol J 14:2134-2146

Liu S, Hua L, Dong S, Chen H, Zhu X, Jiang J, Zhang F, Li Y, Fang X, Chen F (2015) OsMAPK6, a mitogen-activated protein kinase, influences rice grain size and biomass production. Plant J 84:672-681

Mao H, Sun S, Yao J, Wang C, Yu S, Xu C, Li X, Zhang Q (2010) Linking differential domain functions of the GS3 protein to natural variation of grain size in rice. Proc Natl Acad Sci U S A 107:19579-19584

Mori M, Nomura T, Ooka H, Ishizaka M, Yokota T, Sugimoto K, Okabe K, Kajiwara H, Satoh K, Yamamoto K, Hirochika H, Kikuchi S (2002) Isolation and characterization of a rice dwarf mutant with a defect in brassinosteroid biosynthesis. Plant Physiol 130:1152-1161

Qi P, Lin YS, Song XJ, Shen JB, Huang W, Shan JX, Zhu MZ, Jiang L, Gao JP, Lin HX (2012) The novel quantitative trait locus GL3.1 controls rice grain size and yield by regulating Cyclin-T1;3. Cell Res 22:1666-1680

Qiao Y, Piao R, Shi J, Lee S, Jiang W, Kim BK, Lee J, Han L, Ma W, Koh HJ (2011) Fine mapping and candidate gene analysis of dense and erect panicle 3 , DEP3, which confers high grain yield in rice (Oryza sativa L.). Theor Appl Genet 122:1439-1449

Ramkumar G, Sivaranjani AKP, Pandey MK, Sakthivel K, Shobha Rani N, Sudarshan I, Prasad GSV, Neeraja CN, Sundaram RM, Viraktamath BC, Madhav MS (2010) Development of a PCR-based SNP marker system for effective selection of kernel length and kernel elongation in rice. Mol Breed 26:735-740

Sakamoto T, Morinaka Y, Ohnishi T, Sunohara H, Fujioka S, Ueguchi-Tanaka M, Mizutani M, Sakata K, Takatsuto S, Yoshida S (2006) Erect leaves caused by brassinosteroid deficiency increase biomass production and grain yield in rice. Nat Biotechnol 24:105-109

Shan Q, Wang Y, Li J, Zhang Y, Chen K, Liang Z, Zhang K, Liu J, Xi JJ, Qiu J-L, Gao C (2013) Targeted genome modification of crop plants using a CRISPR-Cas system. Nat Biotechnol 31:686-688

Shimin X, Zhou M, Chen S, Zhang B, Schwessinger D (2014) OsSERK1 regulates rice development but not immunity to Xanthomonas oryzae pv. oryzae or Magnaporthe oryzae. J Integr Plant Biol 56:1179-1192

Shomura A, Izawa T, Ebana K, Ebitani T, Kanegae H, Konishi S, Yano M (2008) Deletion in a gene associated with grain size increased yields during rice domestication. Nat Genet 40:1023-1028

Si L, Chen J, Huang X, Gong H, Luo J, Hou Q, Zhou T, Lu T, Zhu J, Shangguan Y, Chen E, Gong C, Zhao Q, Jing Y, Zhao Y, Li Y, Cui L, Fan D, Lu Y, Weng Q, Wang Y, Zhan Q, Liu K, Wei X, An K, An G, Han B (2016) OsSPL13 controls grain size in cultivated rice. Nat Genet 48:447-456

Song XJ, Huang W, Shi M, Zhu MZ, Lin HX (2007) A QTL for rice grain width and weight encodes a previously unknown RING-type E3 ubiquitin ligase. Nat Genet 39:623-630

Suzaki T, Sato M, Ashikari M, Miyoshi M, Nagato Y, Hirano HY (2004) The gene FLORAL ORGAN NUMBER1 regulates floral meristem size in rice and encodes 
a leucine-rich repeat receptor kinase orthologous to Arabidopsis CLAVATA1. Development 131:5649-5657

Tanabe S, Ashikari M, Fujioka S, Takatsuto S, Yoshida S, Yano M, Yoshimura A, Kitano H, Matsuoka M, Fujisawa Y, Kato H, Iwasaki Y (2005) A novel cytochrome P450 is implicated in brassinosteroid biosynthesis via the characterization of a rice dwarf mutant, dwarf11, with reduced seed length. Plant Cell 17:776-790

Tanaka A, Nakagawa H, Tomita C, Shimatani Z, Ohtake M, Nomura T, Jiang CJ, Dubouzet JG, Kikuchi S, Sekimoto H, Yokota T, Asami T, Kamakura T, Mori M (2009) BRASSINOSTEROID UPREGULATED1, encoding a helix-loop-helix protein, is a novel gene involved in brassinosteroid signaling and controls bending of the lamina joint in rice. Plant Physiol 151:669-680

Tong H, Jin Y, Liu W, Li F, Fang J, Yin Y, Qian Q, Zhu L, Chu C (2009) DWARF AND LOW-TILLERING, a new member of the GRAS family, plays positive roles in brassinosteroid signaling in rice. Plant J 58:803-816

Tong H, Liu L, Jin Y, Du L, Yin Y, Qian Q, Zhu L, Chu C (2012) DWARF AND LOWTILLERING acts as a direct downstream target of a GSK3/SHAGGY-like kinase to mediate brassinosteroid responses in rice. Plant Cell 24:2562-2577

Wang L, Xu YY, Ma QB, Li D, Xu ZH, Chong K (2006) Heterotrimeric G protein alpha subunit is involved in rice brassinosteroid response. Cell Res 16:916-922

Wang L, Xu Y, Zhang C, Ma Q, Joo SH, Kim SK, Xu Z, Chong K (2008) OsLIC, a Novel CCCH-Type Zinc Finger Protein with Transcription Activation, Mediates Rice Architecture via Brassinosteroids Signaling. PLoS One 3:e3521

Wang S, Wu K, Yuan Q, Liu X, Liu Z, Lin X, Zeng R, Zhu H, Dong G, Qian Q, Zhang G, Fu X (2012) Control of grain size, shape and quality by OsSPL16 in rice. Nat Genet 44:950-954

Weng J, Gu S, Wan X, Gao H, Guo T, Su N, Lei C, Zhang X, Cheng Z, Guo X, Wang J, Jiang L, Zhai H, Wan J (2008) Isolation and initial characterization of GW5, a major QTL associated with rice grain width and weight. Cell Res 18:1199-1209

Wu Y, Fu Y, Zhao S, Gu P, Zhu Z, Sun C, Tan L (2016a) CLUSTERED PRIMARY BRANCH 1, a new allele of DWARF11, controls panicle architecture and seed size in rice. Plant Biotechnol J 14:377-386

Wu Y, Wang Y, Mi XF, Shan JX, Li XM, Xu JL, Lin HX (2016b) The QTL GNP1 Encodes GA20ox1, Which Increases Grain Number and Yield by Increasing Cytokinin Activity in Rice Panicle Meristems. PLoS Genet 12:e1006386

Xu C, Liu Y, Li Y, Xu X, Xu C, Li X, Xiao J, Zhang Q (2015) Differential expression of GS5 regulates grain size in rice. J Exp Bot 66:2611-2623

Yamamuro C, Ihara Y, Wu X, Noguchi T, Fujioka S, Takatsuto S, Ashikari M, Kitano H, Matsuoka M (2000) Loss of function of a rice brassinosteroid insensitive1 homolog prevents internode elongation and bending of the lamina joint. Plant Cell 12:1591-1606

Yan CJ, Zhou JH, Yan S, Chen F, Yeboah M, Tang SZ, Liang GH, Gu MH (2007) Identification and characterization of a major QTL responsible for erect panicle trait in japonica rice (Oryza sativa L.). Theor Appl Genet 115:1093-1100

Zhang LY, Bai MY, Wu J, Zhu JY, Wang H, Zhang Z, Wang W, Sun Y, Zhao J, Sun X, Yang H, Xu Y, Kim SH, Fujioka S, Lin WH, Chong K, Lu T, Wang ZY (2009) Antagonistic $\mathrm{HLH} / \mathrm{bH} \mathrm{LH}$ transcription factors mediate brassinosteroid regulation of cell elongation and plant development in rice and Arabidopsis. Plant Cell 21:3767-3780

Zhang X, Wang J, Huang J, Lan H, Wang C, Yin C, Wu Y, Tang H, Qian Q, Li J (2012) Rare allele of OSPPKL1 associated with grain length causes extra-large grain and a significant yield increase in rice. Proc Natl Acad Sci U S A 109:21534-21539

Zheng J, Zhang Y, Wang C (2015) Molecular functions of genes related to grain shape in rice. Breed Sci 65:120-126

Zuo J, Li J (2014) Molecular genetic dissection of quantitative trait loci regulating rice grain size. Annu Rev Genet 48:99-118

\section{Submit your manuscript to a SpringerOpen ${ }^{\circ}$ journal and benefit from:}

- Convenient online submission

- Rigorous peer review

- Open access: articles freely available online

- High visibility within the field

- Retaining the copyright to your article

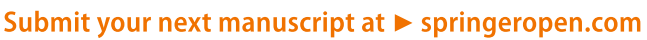

